PROCEEDINGS OF THE

AMERICAN MATHEMATICAL SOCIETY

Volume 132, Number 10, Pages 2951-2958

S 0002-9939(04)07428-3

Article electronically published on June 2, 2004

\title{
ON THE LIFT-OFF CONSTANT FOR ELASTICALLY SUPPORTED PLATES
}

\author{
R. F. BASS, J. HORÁK, AND P. J. MCKENNA \\ (Communicated by David S. Tartakoff)
}

\begin{abstract}
In this paper we continue the study begun by Kawohl and Sweers of the precise constant at which the elastic foundation supporting a bending plate can allow lift-off in the case of downward loading. We provide a number of numerical results and a rigorous result on a different counterexample than the one suggested in Kawohl and Sweers (2002). Important open problems are summarized at the conclusion.
\end{abstract}

\section{INTRODUCTION}

For many years, the equation

$$
\Delta^{2} u=f(x) \text { in } \Omega
$$

has been studied as a model of the deflection of a plate shaped like a region $\Omega$ in $\mathbb{R}^{2}$ under a load $f(x)$.

How the plate is supported at the boundary determines the boundary conditions. If the plate is clamped at the boundary, the conditions are

$$
u=\frac{\partial u}{\partial n}=0 \quad \text { on } \partial \Omega \text {. }
$$

On the other hand, if the plate is simply supported at the boundary (analogous to hinged in one dimension), then the appropriate boundary conditions are

$$
u=\Delta^{2} u=0 \quad \text { on } \partial \Omega \text {. }
$$

The clamped boundary conditions have been the object of much study, centered around the Boggio-Hadamard conjecture (1908), [3, 4, 13, 14], which suggested the physically intuitive result that if $\Omega$ is convex, then a positive load $f(x)$ should result in a positive deflection $u(x)$. This proved not to be the case, with counterexamples by Duffin, Garabedian, and Loewner, [9, 10, 11]. The study of the general properties of $\Delta^{2}$ with clamped boundary conditions continues to be an area of active research, [12.

Received by the editors January 7, 2003

2000 Mathematics Subject Classification. Primary 35J40; Secondary 60 J65.

Key words and phrases. Lift-off constant, vibrating plate, beam, Brownian motion.

The research of the first author was partially supported by NSF grant DMS-9988496.

(C)2004 American Mathematical Society 
One could be tempted to think there is nothing of interest in the case of simply supported boundary conditions. After all, it is a simple example of the maximum principle that if $u$ solves equation (1.1) with (1.3), then $f>0$ implies $u>0$ (since $\Delta v=f$ implies $v<0$, and $\Delta u=v$ ). However, even in one dimension, the situation is not so simple. For example, in civil engineering, one frequently studies the case of a simply supported beam resting on a spring-like foundation. (Railroad engineers model soil as resisting compression by Hooke's law in this way, calling the foundation a Winkler foundation, [16.) This results in an equation

$$
u^{i v}+b u=f(x)
$$

on an interval $(0, L)$. Here, the constant $b$ represents the stiffness of the resistance of the soil to compression. Then, the question of whether the linear operator with hinged-end boundary conditions is positivity-preserving (denoted P-P) is equivalent to asking if the plate lifts off its foundation under any load $f$. The result is surprising: if the spring constant is too big, the beam can lift off, with positive downward loads $f$ resulting in negative (upward) solutions $u$.

Specifically, it was shown in [19, see also [15], that on the interval $(0, L)$, there is a constant $b_{L O}(L)$ such that if $b>b_{L O}(L)$, then lift-off occurs in (1.4), whereas if $b<b_{L O}(L)$, then the equation is P-P and no lift-off occurs. The method consists of explicitly calculating the Green's function for the interval $(0,1)$, and showing that it changes sign when $b>950.884$.

It was only quite recently that the analogous question was asked for the multidimensional case. In [17, the question of estimating the best constant $b_{L O}(\Omega)$ such that if $b<b_{L O}(\Omega)$, then the equation

$$
\Delta^{2}+b u=f(x)
$$

is P-P was addressed. In addition, it was conjectured that among regions of given area, the largest $b_{L O}(\Omega)$ should occur for a ball. The reality proved considerably more complicated.

The first significant progress on this question occurs in a paper by Kawohl and Sweers, [15. Here they prove a series of new results on the multi-dimensional case. Perhaps most important, they show that the set of $b$ for which equation (1.5) is $\mathrm{P}-\mathrm{P}$ is an interval. A counterexample is given to the aforementioned conjecture, with an extremely non-convex domain. It is also shown that if $\Omega$ is a rectangle of sides $L$ and $1 / L$, then $\liminf _{L \rightarrow \infty} b_{L O}(\Omega) \geq \pi^{2}$.

The method employed in this paper was also interesting. Instead of attacking the equation directly, the authors estimate $b_{L O}(\Omega)$ from below by estimating instead the positivity-preserving properties of a different system:

$$
\begin{array}{r}
-\Delta u=f-\lambda v \text { in } \Omega, \\
-\Delta v=f \text { in } \Omega, \\
u=v=0 \text { on } \partial \Omega .
\end{array}
$$

They show that there is a best possible constant $\lambda_{c}(\Omega)$ such that the system (1.6) is P-P in $f$ if $\lambda \leq \lambda_{c}(\Omega)$. They also show that $\lambda_{c}(\Omega)^{2} \leq b_{L O}(\Omega)$. They then use techniques from probability to estimate $\lambda_{c}(\Omega)$ using the fact that

$$
\lambda_{c}(\Omega)=\left(\mathbb{E}_{x}^{y}\left(\tau_{\Omega}\right)\right)^{-1}
$$


where $\mathbb{E}_{x}^{y}\left(\tau_{\Omega}\right)$ is the expectation for the lifetime of Brownian motion that is conditioned to start at $x$, converge to $y$, and be killed when reaching the boundary of $\Omega$. They then used some estimates for $\mathbb{E}_{x}^{y}\left(\tau_{\Omega}\right)$, which occur first in two dimensions in [6] and for three or more dimensions in [7].

In this paper, we continue their investigation in two ways. First, we shall use the finite element package from MATLAB to perform numerical experiments to estimate $b_{L O}(\Omega)$ for a variety of different geometries.

Second, we give a new counterexample with new probabilistic proof for a shape that at least to us seems more intuitive than the one presented in 15. Since the original example was considered somewhat surprising in probability circles when first published, we believe that it is interesting that this new shape should occur so naturally in this new context.

These calculations lead to many interesting unsolved problems, which we will summarize in the last section.

\section{NumericAl EVIDENCE}

In this section, we summarize what we have been able to conclude from numerical experiments. The idea is fairly crude but effective. Fix a region $\Omega \subset \mathbb{R}^{2}$ such that $|\Omega|=1$. Fix a value for $b$ in equation (1.5). Impose a triangulated grid on the region. Impose a point load at one of the points on the grid. (This plays the role of $f$.) Then check the resulting solution $u(x)$ of $(1.5)$ at all points of the grid to see whether $u$ changes sign. Loop over all points of the grid. If $u$ never changes sign, then we can conclude, modulo numerical errors, that $b<b_{L O}(\Omega)$. Otherwise, $b>b_{L O}(\Omega)$.

Use a bisection algorithm to estimate $b_{L O}(\Omega)$. Finally, repeat this huge loop on a refined mesh to reduce the probability of numerical error. If the numbers remain stable under repeated refinement, we can have some confidence that the results are reasonable. Now to the results.

2.1. Disc of unit area. In the case of the disc, originally conjectured to give the highest possible constant, we have $b_{L O}\left(\Omega_{L}\right) \approx 1045.9$. The best place to apply the point load, to first see the lift-off, is as near as one can get to the circumference.

2.2. Rectangles. Our next experiment is on the class of unit rectangles $\Omega_{L}=$ $[0, L] \times[0,1 / L]$. In this case, the largest value of $b_{L O}\left(\Omega_{L}\right)$ occurs for the square, $L=1$. The value is approximately 884.6. In terms of orders of magnitude, this is consistent with the calculated value of the unit interval in one dimension.

So far, this is consistent with the conjecture. The only other analytic information we have is that $\liminf _{L \rightarrow \infty} b_{L O}\left(\Omega_{L}\right) \geq \pi^{2}$, [15].

Figure 1 shows the values of $b_{L O}\left(\Omega_{L}\right)$ as graphed. Note that the lower bound $\pi^{2}$ appears to be unrealistically low. Two natural questions arise at this point: first, can one find an analytic expression for the curve? Second, can one give a better lower bound for $\liminf _{L \rightarrow \infty} b_{L O}\left(\Omega_{L}\right)$ ?

We also observed in the course of these calculations that the "best" point for imposing the point mass in order to get lift-off was at the corners.

Thus far, it might seem that the conjecture is still intact for convex domains. After all, the most symmetric region is the square, and this gives the biggest liftoff constant. The next subsection investigates some other shapes and gives an interesting counterexample. 


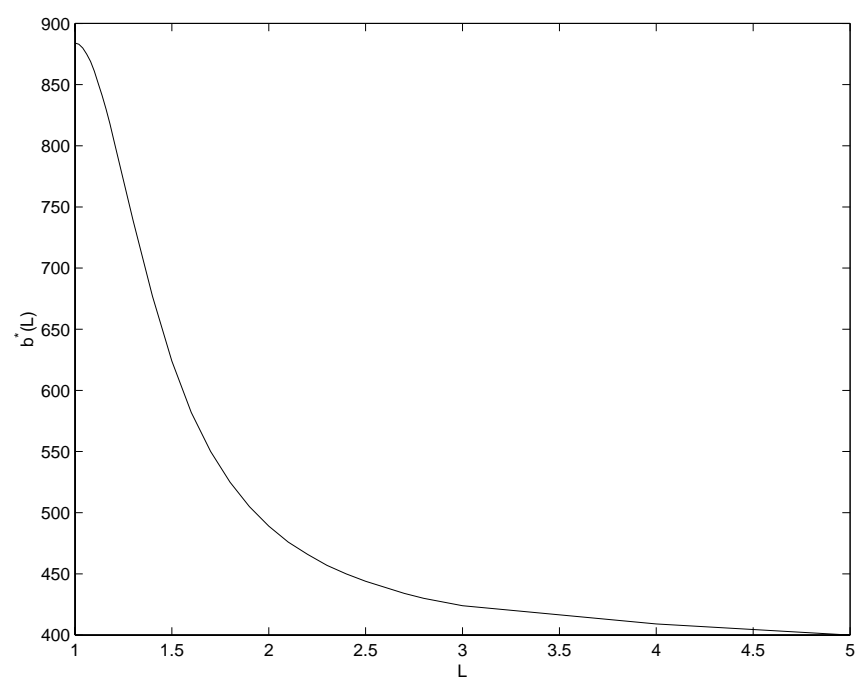

Figure 1. Values of $b_{L O}$ for $\Omega_{L}=[0, L] \times[0,1 / L]$.

2.3. Other shapes and a counterexample. The first and most surprising new shape is the result for the pentagon. In the case of this geometry, the figure was triangulated and a point load was applied at the center of each triangle. We found that the best place to impose the load was at the side of the pentagon, at approximately one eighth of its length. The lift-off constant thus obtained was $b_{L O}(\Omega) \approx 1073.1$. Of course, this is significantly higher than the value for the circle. Further refinements of the grid did not significantly change this constant.

This result leaves very much open the question of what shape will give the best lift-off constant. The natural question is how this compares with other regular polygons. Therefore, we checked the case of the regular hexagon and octagon. For the hexagon of unit area, we obtained a lift-off constant of $b_{L O}(\Omega) \approx 993.2$ and for the octagon, $b_{L O}(\Omega) \approx 1022.1$. In each case, the point to apply the load was at a corner, and the lift-off occurred at the opposite side.

For completeness, we also did the calculation for a right-angle triangle with sides of lengths 1 and 2 . Here the constant was $b_{L O}(\Omega) \approx 532.2$.

Thus these calculations leave us with no clear intuition as to what convex shape will give the largest lift-off constant. It is also unclear, given any shape, how to characterize the point at which the applied load gives first lift-off. Presumably, it is always at the boundary but sometimes at a corner and sometimes at a straight edge.

As shown in [15], from probability, one can obtain a shape of measure one such that $b_{L O}(\Omega)$ can be as large as desired. Our work with the long thin rectangle gave us the insight to construct a different example.

The central idea of this new counterexample to the (nonconvex) McKenna-Walter conjecture (now of course totally in ruins) is that a small long thin rectangle produces a lift-off constant that goes to $\infty$. Thus it is natural to assemble a comb-like geometry, in which each segment is a very small thin rectangle linked together so that the only way to produce lift-off in one segment is to go round a corner of 
the comb. Such an example is shown in Figure 2 (d). The lift-off constant was estimated at a relatively large $b_{L O}(\Omega) \approx 2993.9$.

(a)

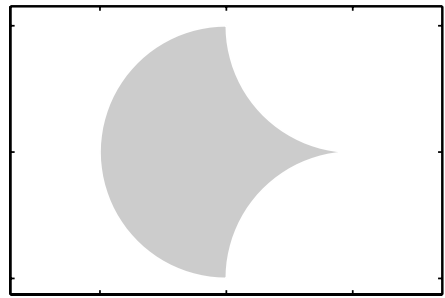

(c)

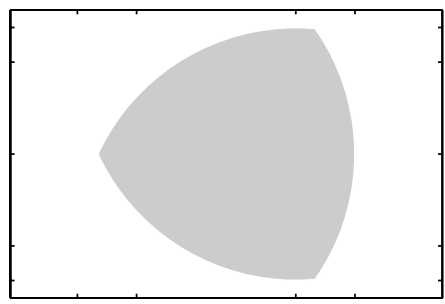

(b)

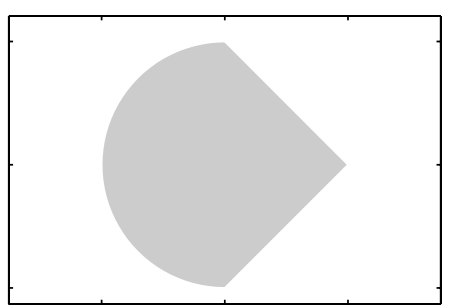

(d)

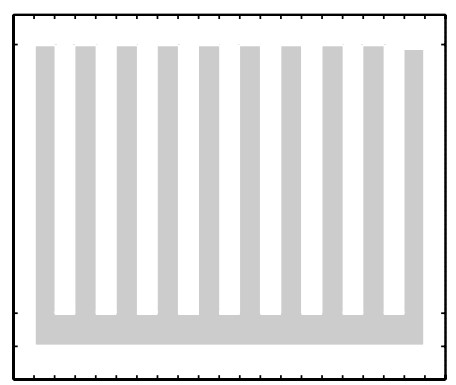

FiguRE 2. Various shapes of domains $\Omega$

\begin{tabular}{|l|c|}
\hline Shape of the domain $\Omega$ & computed $b_{L O}(\Omega)$ \\
\hline Equilateral triangle & 917.9 \\
Square & 884.6 \\
Regular pentagon & 1073.1 \\
Regular hexagon & 993.2 \\
Regular octagon & 1022.1 \\
Disk & 1045.9 \\
Right-angle triangle, sides 1 and 2 & 532.2 \\
Shape in Figure 2 (a) & 760.6 \\
Shape in Figure 2 (b) & 919.8 \\
Shape in Figure 2 (c) & 1044.5 \\
Comb-Figure 2 (d) & 2993.9 \\
\hline
\end{tabular}

TABLE 1. Approximate values of $b_{L O}$ for various domains $\Omega \subset \mathbb{R}^{2}$ with unit area 


\section{A Rigorous PROOF FOR LIFT-OFF FOR THE COMB}

As remarked in the introduction, we shall estimate $b_{L O}(\Omega)$ from below by estimating $\tau$ as the lifetime of Brownian motion killed on exiting $D-\{y\}$. Let

$$
\Gamma(D)=\sup _{x, y \in D} \mathbb{E}_{y}^{x} \tau
$$

be the supremum of the expected lifetime of Brownian motion started at $x$ and conditioned to go to $y$.

Saying the boundary Harnack principle holds in a domain $D_{0}$ means the following. Given a compact set $K$ contained in an open set $V$ and $x_{0} \in V$, there exists $c_{0}$ such that if $u$ and $v$ are positive harmonic functions in $D_{0}$ that vanish continuously on $V \cap \partial D_{0}$, then

$$
\frac{u(x)}{v(x)} \leq c_{0} \frac{u\left(x_{0}\right)}{v\left(x_{0}\right)}, \quad x \in K \cap D_{0} .
$$

We know that a boundary Harnack principle holds in Lipschitz domains. Since we are in two dimensions, an easy conformal mapping argument shows that a boundary Harnack principle also holds when the domain is a disk minus two line segments.

Theorem 1. Given $\epsilon>0$ there exists a subdomain $D$ of $(0,1)^{2}$ such that $(0,1)^{2}-D$ has Lebesgue measure 0 but $\Gamma(D)<\epsilon$.

Proof. Let $L>0$ be an integer, let $E_{i}=\left\{\left(x_{1}, x_{2}\right): x_{1}=i / L, 0<x_{2}<1-(1 / L)\right\}$, and let

$$
D=(0,1)^{2}-\bigcup_{i=1}^{L-1} E_{i} .
$$

We call $D$ a comb. Clearly the Lebesgue measure of $(0,1)^{2}-D=0$, and we need to show that $\Gamma(D)$ will be small if $L$ is large.

Let

$$
F_{i}=\left\{\left(x_{1}, x_{2}\right): i / L<x_{1}<(i+1) / L, 0<x_{2}<1-\sqrt{1 / L}\right\} .
$$

Let $g(x, y)$ be the Green function for $D$. Set

$$
g_{y}(x, z)=\frac{g(x, z) g(z, y)}{g(x, y)}
$$

so that $g_{y}(x, z)$ is the Green function for Brownian motion in $D-\{y\}$ conditioned to exit at $y$. Then

By [2, we have

$$
\mathbb{E}_{y}^{x} \tau=\int_{D} g_{y}(x, z) d z
$$

$$
g_{y}(x, z) \leq c_{1}\left[1+\log ^{+}(1 /|x-z|)+\log ^{+}(1 /|y-z|)\right],
$$

where $c_{1}$ does not depend on $L$.

Fix $x$ and $y$ in $D$. Applying the boundary Harnack principle to $g_{y}(x, z)$ in appropriate subdomains, we may without loss of generality suppose that $x_{1} \neq i / L$ for any $i$ and similarly for $y$. If $x \in F_{i}$ for some $i$, let $i_{x}$ be the value of $i$ for which this holds. If $x$ is not in any $F_{i}$, let $i_{x}$ be the value of $i$ for which $x-(0,1 / 2)$ is in $F_{i}$. We define $i_{y}$ similarly. We know that when $L$ is large, the Lebesgue measure of $C=\left\{\left(x_{1}, x_{2}\right): 0<x_{1}<1,1-\sqrt{1 / L}<x_{2}<1\right\}$ is small. Using (3.2), the integral of $g_{y}(x, z)$ over $C \cup\left(\bigcup_{j=-2,+2}\left(F_{i_{x}+j} \cup F_{i_{y}+j}\right)\right)$ can be made as 
small as we like by taking $L$ large. We complete the proof by showing that if $z \notin C \cup\left(\bigcup_{j=-2,+2}\left(F_{i_{x}+j} \cup F_{i_{y}+j}\right)\right)$, then $g_{y}(x, z)$ will be small when $L$ is large.

Suppose $z \in F_{i}$, and let $x_{i}=\left(\frac{2 i+1}{2 L}, 1-\frac{2}{L}\right)$ and $y_{i}=\left(\frac{2 i+1}{2 L}, 1-\frac{7 / 4}{L}\right)$. Let $T$ be the first time that conditioned Brownian motion $W_{t}$ hits the line segment $\left\{y=1-\frac{2}{L}\right\} \cap F_{i}$. By the strong Markov property and the fact that $g_{y}$ is harmonic for conditioned Brownian motion as long as we are away from $y$ and $z$,

$$
g_{y}(x, z) \leq \mathbb{E}^{x} g_{y}\left(W_{T}, z\right) .
$$

By the boundary Harnack principle applied in the ball of radius $2 / L$ centered at $x_{i}$ intersected with $D, \mathbb{E}^{x} g_{y}\left(W_{T}, z\right)$ is bounded by a constant times $g_{y}\left(x_{i}, z\right)$. Now $g_{y}\left(x_{i}, z\right)=g_{x_{i}}(y, z)$ is also the Green function for Brownian motion started at $y$ and conditioned to exit $D-\left\{x_{i}\right\}$ at $x_{i}$. So using the strong Markov property and the boundary Harnack principle and arguing as above, it suffices to obtain a bound on $g_{x_{i}}\left(y_{i}, z\right)$.

Now $g\left(x_{i}, y_{i}\right)$ is larger than the Green function for $B\left(x_{i}, L / 2\right)$ evaluated at $x_{i}$ and $y_{i}$, and by scaling this is larger than $c_{2}$. Let $S$ be the first time that Brownian motion hits the ball of radius $L / 4$ about $x_{i}$. If $w$ is on the boundary of this ball, then $g\left(w, x_{i}\right)$ is less than the Green function for the ball of radius 2 centered at $x_{i}$ evaluated at $x_{i}$ and $w$, and so $g\left(w, x_{i}\right) \leq c_{2} \log (1 / L)$. By the strong Markov property and the fact that $g$ is harmonic away from $x_{i}$,

$$
g\left(z, x_{i}\right) \leq \mathbb{E}^{z}\left(g\left(W_{S}, x_{i}\right) ; S<\tau\right) \leq c_{2} \log (1 / L) \mathbb{P}^{z}(S<\tau),
$$

and similarly for $g\left(y, z_{i}\right)$. But $F_{i}$ is a strip of width $1 / L$ and the distance between $x$ and $z_{i}$ is at least $c_{3} / \sqrt{1 / L}$. Using Lemma III.1.5 of [1],

$$
g\left(x, z_{i}\right) \leq c_{4} \log (1 / L) e^{-c_{5} \frac{1}{\sqrt{L}} / \frac{1}{L}} .
$$

We have a similar estimate for $g\left(y, z_{i}\right)$. This, of course, can be made as small as we like by taking $L$ large enough.

\section{Conclusions and open Questions}

There remain several interesting open questions about which we have little intuition at present.

The most compelling of these is the question of the convex shape of area one that will give the largest lift-off constant. Clearly, the original conjecture, which might have survived the counterexample of [15] is now in ruins, and it is not clear what would make a compelling replacement. More computational experiments are in order.

A second natural question is how to give better rigorous estimates for the liftoff constant from below. The existing method of Kawohl and Sweers gives lower estimates which are indeed very low. For example, for the long thin rectangle, Kawohl and Sweers can prove the probabilistic estimate that in the infinite limit, the curve in Figure 1 is bounded below by $\pi^{2}$, while the computational evidence suggests something of the order of 400.

A third pressing need is to find upper bounds for the lift-off constant. So far, nothing is known. It should be possible to provide estimates in terms of the inradius of the domain $\Omega$. We can prove upper bounds on $\lambda_{c}(\Omega)$ in terms of the in-radius, but do not know if this implies anything about upper bounds for $b_{L O}(\Omega)$. 
Finally, it may also be possible to say more about the best point to apply a load in the region $\Omega$ in order to induce lift-off for values of $b$ close to $b_{L O} \Omega$. It seems clear that such a point should be at the boundary, though where at the boundary is not clear.

\section{REFERENCES}

[1] Bass, R. F., Probabilistic Techniques in Analysis, Springer-Verlag, New York, 1995. MR 96e:60001

[2] Bass, R. F., and Burdzy, K., Conditioned Brownian motion in planar domains, Probab. Theory Related Fields 101 (1995) 479-493. MR 96b:60199

[3] Boggio, T., Sull'equilibrio delle piastre elastiche incastrate, Rend. Acc. Lincei 10 (1901), 197205.

[4] Boggio, T., Sulle funzioni di Green d'ordine $m$, Rend. Circ. Mat. Palermo 20 (1905), 97-135.

[5] Cranston, M.; Fabes, E.; and Zhao, Z., Potential theory for the Schrödinger equation, Bull. Amer. Math. Soc. (N.S.) 15 (1986), no. 2, 213-216. MR 88d:60197

[6] Cranston, M. and McConnell, T. R., The lifetime of conditioned Brownian motion. Z. Wahrsch. Verw. Gebiete 65 (1983), no. 1, 1-11. MR 85d:60150

[7] Cranston, M., Lifetime of conditioned Brownian motion in Lipschitz domains. Z. Wahrsch. Verw. Gebiete 70 (1985), no. 3, 335-340. MR 87a:60088

[8] Coffman, C. V., On the structure of solutions to $\Delta^{2} u=\lambda u$ which satisfy the clamped plate condition on a right angle, SIAM J. Math. Anal. 13, 746-757 (1982). MR 84a:35015

[9] Coffman, C. V. and Duffin, R. J., On the structure of biharmonic functions satisfying the clamped plate conditions on a right angle, Adv. Appl. Math. 1 (1980), 373-389. MR 82e:31004

[10] Duffin, R. J., On a question of Hadamard concerning super-biharmonic functions, J. Math. Phys. 27 (1949), 253-258. MR 10:534h

[11] Garabedian, P. R., A partial differential equation arising in conformal mapping, Pacific J. Math. 1 (1951), 485-524. MR 13:735a

[12] Grunau, H.-C. and Sweers, G., Positivity for perturbations of polyharmonic operators with Dirichlet boundary conditions in two dimensions. Math. Nachr. 179 (1996), 89-102. MR 97f:35040

[13] Hadamard, J., Mémoire sur le problème d'analyse relatif à l'équilibre des plaques élastiques incastrées, Mémoires presentes par divers savants à l'Académie des Sciences, Vol. 33 (1908), $1-128$.

[14] Hadamard, J., Sur certains cas intéressants du problème biharmonique, Atti IVe Congr. Intern. Mat. Rome (1909), 12-14.

[15] Kawohl, B., and Sweers, G., On "anti"-eigenvalues for elliptic systems and a question of McKenna and Walter, Indiana Univ. Math. J. 51 (2002), no. 5, 1023-1040. MR 2003j:35059

[16] Lin, Li, and Adams, G. G., Beam on tensionless elastic foundation J. Eng. Mech., 113 (1986), 542-553.

[17] McKenna, P. J., and Walter, W., Nonlinear oscillations in a suspension bridge, Arch. Rational Mech. Anal., 98 (1987), 167-177. MR 88a:35160

[18] Patil, S. P., Response of infinite railroad track to vibrating mass, J. Eng. Mech., 114 (1988) 688-703.

[19] Schröder, Johann, Operator inequalities, Mathematics in Science and Engineering, 147, Academic Press, Inc. [Harcourt Brace Jovanovich, Publishers], New York-London, 1980. MR $84 \mathrm{k}: 65063$

Department of Mathematics, University of Connecticut, Storrs, Connecticut 06269

E-mail address: bass@math.uconn.edu

Department of Mathematics, University of Basel, Basel, Switzerland

Current address: Mathematisches Institut, Universität zu Köln, Weyertal 86-90, D-50923 Köln, Germany

E-mail address: jhorak@math.uni-koeln.de

Department of Mathematics, University of Connecticut, Storrs, Connecticut 06269

E-mail address: mckenna@math.uconn.edu 